\title{
Lowering Gestational Diabetes Risk by Prenatal Weight Gain Counseling
}

\author{
Evelyn M. Figueroa, MD, Kara Nitti, MPH, and Stephen M. Sladek, MD
}

Purpose: Excess weight gain during pregnancy is at epidemic proportions, and pregnancy complications are also on the rise. We sought to determine whether better weight gain counseling of expectant mothers will improve obstetric outcomes.

Methods: Our historic control study design included 2 years of preintervention data, then 6 months of physician and staff training in prenatal weight gain counseling in accordance with 2009 Institute of Medicine guidelines, and finally, 2 more years of data collection for postintervention outcomes. Seven family medicine residency clinics monitored 1571 continuity prenatal cases. Counseling recommendations were noted and the following outcomes were analyzed: gestational age, birth weight, route of delivery, and the incidences of hypertension and gestational diabetes. Multiple logistic regression was used to control for demographic variables and body mass index at enrollment.

Results: Institute of Medicine congruent counseling increased from $10 \%$ to $63 \%(P<.01)$. Excess weight gain decreased from $46.4 \%$ to $41.5 \%$ (adjusted odds ratio $[\mathrm{AOR}]=0.85 ; 95 \% \mathrm{CI}, 0.63-1.16$; $P=.10)$. Gestational diabetes decreased significantly from $11.5 \%$ to $7.3 \%(P=.008)$. The difference remained statistically significant even after adjusting for prepregnancy obesity and other clinical and demographic characteristics $(A 0 R=0.54 ; 95 \% C I, 0.32-0.91 ; P=.02)$. Differences in gestational age, birth weight, hypertension, primary cesarean, and shoulder dystocia were not statistically significant.

Conclusions: Improved weight gain counseling of prenatal patients by physicians did reduce the pregnancy complication of gestational diabetes. This occurred even though the trend toward less excess weight gain was not statistically significant. (J Am Board Fam Med 2020;33:189-197.)

Keywords: Counseling, Gestational Diabetes, Gestational Weight Gain, Historically Controlled Study, Hypertension, Logistic Models, Obstetrics, Pregnancy Complications

Weight gain and nutrition influence the health of both mothers and their offspring. The prenatal period presents an opportunity to guide women in lifestyle changes because of frequent visits, motivation to avoid delivery complications, and a strong desire to have a healthy baby. ${ }^{1}$ Despite this

This article was externally peer reviewed.

Submitted 6 June 2019; revised 08 September 2019; accepted 11 September 2019.

From the Department of Family Medicine, University of Illinois, Chicago (EVF); Advocate Research Institute, Oaklawn, IL (KN); Advocate Christ Family Medicine Residency, Oak Lawn, IL (SMS).

Funding: Society of Teachers of Family Medicine (STFM) research grant $(\$ 5,000)$. This funding source had no involvement in study design; in the collection, analysis, nor interpretation of data; in the writing of the report; nor in the decision to submit the article for publication. Indirect costs were covered by each study site.

Conflicting of interests: none.

Corresponding author: Stephen M. Sladek, MD, Advocate Christ Family Medicine Residency, Oak Lawn, IL (E-mail: stephen.sladek@advocatehealth.com). opportunity, excessive weight gain in pregnancy is of epidemic concern in the United States. As of $2008,53 \%$ of normal weight women, $72 \%$ of overweight women, and $83 \%$ of obese women gained excess weight during pregnancy. ${ }^{2}$

Both high prepregnancy body mass index (BMI) and excessive gestational weight gain correlate with obstetric complications. In 2009, the Institute of Medicine (IOM) reviewed the evidence available at the time in the process of updating their guidelines for recommended weight gain during pregnancy. ${ }^{3}$ It was not clear then how much of the increased risk is due to prepregnancy weight versus further weight gain during the pregnancy. New data are rapidly becoming available to shed light on this possible distinction. This is clinically important because standard prenatal care beginning at 8 to 12 weeks of gestation can affect only gestational weight gain, but not prepregnancy BMI.

Several newer studies on prepregnancy BMI clarify the relationship with pregnancy complications. 
In 2015, the British Columbia Perinatal Data registry of 226,958 births reported that $10 \%$ lower prepregnancy BMI was associated with $10 \%$ lower preeclampsia, gestational diabetes, macrosomia, and stillbirth. From $20 \%$ to $30 \%$ lower BMI was required to see lower rates of cesarean, shoulder dystocia, neonatal intensive care admission, and neonatal mortality. ${ }^{4}$ A 2015 cohort study from Beijing, China $(n=14,451)$ confirmed the higher prepregnancy BMI association with higher preeclampsia, gestational diabetes, cesarean, and macrosomia (even after controlling for gestational diabetes). ${ }^{5}$

Not enough is known about the specific effect of excess prenatal weight gain on top of already high prepregnancy BMI. We found 2 major reports suggesting that preventing excessive weight gain during pregnancy has the potential to promote better pregnancy outcomes: both looked at outcomes stratified by prepregnancy BMI to isolate the effect of subsequent prenatal weight gain. The first study was a secondary analysis of a National Institutes of Health trial of vitamin $\mathrm{C}$ and $\mathrm{E}$ to prevent preeclampsia $(n=9969)$ that showed excess weight gain was associated with increased hypertensive disorders, cesarean, and macrosomia. ${ }^{2}$ The second study was a 2017 meta-analysis of 23 studies including 1.3 million mothers with excess prenatal weight gain associated with higher rates of macrosomia and cesarean delivery. ${ }^{6}$

Counseling is challenging because medical prenatal visits are brief and a uniform approach cannot fit all encounters. Advice must be individualized to a mother's culture, values, habitus, and personal circumstances. Decades ago, the focus was on limiting weight gain in all pregnant women. The 2009 IOM guideline provided a consensus that maternal weight gain goals vary depending on initial BMI. Maternity care providers need to know how to both encourage minimum healthy weight gain and prevent excessive weight gain, all while maintaining positive rapport with their patients. ${ }^{3}$

Despite the guideline and possible consequences of excessive weight on pregnancy, health care providers often do not counsel per the recommended guideline because of many perceived barriers. ${ }^{1}$ In Ontario, Canada, only $29 \%$ of prenatal patients received a weight gain prescription from their physician (and only 12\% of the patients met the prescribed weight gain goal). ${ }^{7}$ Nurse practitioners, physicians, and midwives give varying recommendations on the weight gain range they recommend. Some advise a total weight gain amount regardless of prepregnancy BMI, whereas others advise an amount lower than the IOM recommendations. Providers have given many reasons for not adhering to the IOM guidelines, ranging from fear of patient stigmatization to perceived lack of efficacy. ${ }^{1}$

In 2014, when this study was designed, clinical trials to prevent excess weight gain during pregnancy had been few, usually involved diet counseling or physical activity, and showed modest results. In a 2012 meta-analysis of behavioral counseling (3 studies that could be combined, $n=443$ ), intervention lowered excess weight gain, but the confidence interval was wide and crossed 1 (relative risk $[\mathrm{RR}]=0.47$; CI, 0.08-2.85). ${ }^{8}$ A 2015 meta-analysis of 13 articles involving 2500 sedentary women showed that an exercise program decreased pregnancy weight gain by $1.1 \mathrm{~kg}$ and significantly decreased gestational diabetes (from $9.0 \%$ to $4.8 \%$; $\mathrm{RR}=0.69$; CI, 0.52-0.9). ${ }^{9}$ But, that meta-analysis excluded a high-quality randomized trial of exercise for 375 expectant mothers that failed to show a significant decrease in gestational diabests $(7 \%$ in the intervention group vs $6 \%$ for control, $P=.52) .{ }^{10}$

The Family Medicine Midwest Maternity Care Collaborative was assembled to heed the IOM 2009 call to action, and we designed this study. Our hypothesis was that provider and staff education will increase the proportion of prenatal patients being counseled according to the 2009 IOM updated weight gain in pregnancy guideline and will lower excess weight gain. Our secondary goals were to measure the effects, if any, on pregnancy outcomes.

\section{Methods \\ Design}

This was a pre/postintervention study in which we used a 2-year historic time period for the preintervention group (obtained by retrospective chart review 2012-2013), a 6-month clinical educational intervention on proper prenatal weight gain counseling per the 2009 IOM guidelines, and a 2-year prospective postintervention group (about 20152017, beginning whenever a site received their local institutional review board approval of the original protocol approved by the institutional review board at the originating site [University of Illinois, Chicago]). 


\section{Eligibility}

Women with the following characteristics were chose for the study: a singleton pregnancy; maternal age of 15 to 45 years old at time of delivery; initiation of prenatal care by 16 weeks gestation; 6 or more prenatal visits, including 1 visit after 35 weeks; and delivery at the hospital affiliated with the practice. These characteristics were chosen to allow a reasonable opportunity for weight gain counseling during prenatal care to possibly influence maternal weight gain. Exclusion criteria were prepregnancy diabetes, daily prednisone use $>21$ days, or prepregnancy hypertensive disease requiring $\geq 2$ medications.

\section{Data Collection}

Data collection included demographics (maternal age, race, ethnicity, and insurance type), parity, prepregnancy weight and BMI, as well as total weight gained. We also collected data on the following pregnancy outcomes: date of delivery, gestational age, newborn weight, and the mode of delivery (vaginal delivery, vacuum or forceps, shoulder dystocia during vaginal delivery; and primary or repeat cesarean). Data on complications of pregnancy were collected, including gestational diabetes (2stage testing: 1-hour nonfasting 50 -g oral glucose challenge test and, if $\geq 140 \mathrm{mg} / \mathrm{dL}$, then 3 -hour fasting $100-\mathrm{g}$ oral glucose tolerance testing [Coustan criteria] $)^{11}$ and hypertensive disorders of pregnancy (preeclampsia, eclampsia, and gestational hypertension). ${ }^{12}$

\section{Intervention}

Our 6-month initial educational period involved local site training on the use of the IOM 2009 guidelines $^{3}$ (Table 1). Each site held education sessions with support staff, nurses, residents, faculty, and teaching attending physicians detailing the importance of weight gain counseling in pregnancy, and the sessions also included motivational counseling techniques. ${ }^{13}$ The initial didactic teaching session set the expectation that weight gain should be addressed with mothers at every prenatal visit. Site principal investigators (residency faculty) were "champions" to constantly remind providers to follow this dictate and the 2009 IOM guideline throughout the entire 2-year postintervention time period. Patient handouts and brochures to help reiterate this education were provided, but their use was optional (Appendix 1). Some sites were able to provide electronic medical record weight gain counseling prompts. Six months were allowed to pass after the educational sessions before recording weight gain and pregnancy outcomes so as to give time for the new awareness to possibly influence the new group of prenatal patients entering the practice.

\section{Data Analyses}

All eligible subjects in both groups were included, as we performed an intention-to-treat analysis. A "per protocol" analysis was done only for exploratory purposes. Descriptive statistics were calculated for all variables. Group means and standard deviations are presented for continuous variables. Counts and percentages are presented for categorical variables. Comparisons were made between groups using Student's $t$ test and Pearson's $\chi^{2}$ test of proportions as applicable. Adjusted multivariate logistic regression models were constructed for the outcome of IOM guideline-recommended weight gain using backward stepwise elimination. Interactions were assessed between all covariates in the final model (SAS software, version 15.1). All statistical tests were 2 -tailed and a $P$ value of .05 was considered statistically significant in these analyses. Sensitivity analyses were performed by analyzing pre- and postintervention outcomes after omitting the data from single sites with outlying incidences of any parameter.

\section{Results}

A total of 1571 pregnant women were identified during the study period. Of these women, 795 $(50.6 \%)$ were seen during preintervention period and $776(49.4 \%)$ were seen during the postintervention period. The mean maternal ages of each group were comparable, with a mean of $27.5 \pm 5.6$ years in preintervention and 28.0 \pm 5.6 years in postintervention groups; the proportions of insurance types were also comparable (Table 2). However, a significant difference in the proportions of Hispanic mothers was identified, with $26.2 \%$ being Hispanic in the preintervention group compared with $40.3 \%$ being Hispanic in the postintervention group $(P<.01$; Table 2$)$. There was a borderline significant difference in the proportion identified as obese at enrollment, with $27.0 \%$ obese in the preintervention group and $32.5 \%$ obese in the postintervention group $(P=.055$; Table 2$)$. 
Table 1. Institute of Medicine 2009 Pregnancy Weight Gain Guidelines ${ }^{3}$

\begin{tabular}{lcccc}
\hline $\begin{array}{l}\text { Recommendation by Prepregnancy } \\
\text { BMI }\end{array}$ & $\begin{array}{c}\text { Total Weight Gain } \\
\text { (Kg) }\end{array}$ & $\begin{array}{c}\text { Total Weight Gain } \\
\text { (lbs) }\end{array}$ & $\begin{array}{c}2^{\text {nd }} \text { and } 3^{\text {rd }} \text { Trimester } \\
\text { (lbs/week) }\end{array}$ & $\begin{array}{c}2^{\text {nd }} \text { and } 3^{\text {rd }} \text { Trimester } \\
\text { (lbs/month) }\end{array}$ \\
\hline Underweight: $<18.5$ & 13 to 18 & 28 to 40 & 1 to 1.3 & 4 to 6 \\
Normal: 18.5 to 24.9 & 11 to 16 & 25 to 35 & 0.8 to 1.0 & 4 \\
Overweight: 25 to 29.9 & 7 to 11 & 15 to 25 & 0.5 to 0.7 & 2 \\
Obese: $\geq 30$ & 5 to 9 & 11 to 20 & 0.4 to 0.5 & 1.5 to 2 \\
\hline
\end{tabular}

BMI, body mass index.

A significant difference occurred as intended between pre- versus postintervention regarding weight gain counseling: $10.1 \%$ of those in the preintervention group received IOM-congruent counseling compared with $62.8 \%$ of those in the postintervention group $(P<.01$; Table 3$)$.

Total weight gain did decrease between groups: the preintervention group had a mean weight gain of 28.3 pounds $(12.84 \mathrm{~kg})$ compared with 25.9 pounds $(11.75 \mathrm{~kg})$ in the postintervention group. However, this difference did not reach statistical significance $(P=.61)$ (Table 3$)$. Multivariate regression to adjust for any counseling received, site, race, insurance status, parity, pre-BMI, and maternal age again showed a trend toward decrease in maternal prenatal weight gain, but it was still not statistically significant (adjusted odds ratio $[\mathrm{AOR}]=0.85 ; 95 \%$ CI, 0.63-1.16; $P=.10$; Table 4; Appendix 2). Preversus postintervention was not significantly associated with low weight gain $(\mathrm{AOR}=1.2 ; 95 \% \mathrm{CI}$, 0.85-1.69; $P=.31$; Table 4; Appendix 2). Hence, our improved counseling did not go to the overzealous extreme of thwarting a healthy minimum weight gain during pregnancy.

Pre- versus postintervention was significantly associated with a decrease of gestational diabetes $(\mathrm{AOR}=0.54 ; 95 \% \mathrm{CI}, 0.32-0.91 ; P=.02)$ after adjusting for counseling received, site, race, insurance status, parity, pre-BMI, and maternal age (Table 5; Appendix 2).

Table 2. Patient Characteristics at Entry to Prenatal Care* $(n=1571)$

\begin{tabular}{|c|c|c|c|}
\hline Variable & Preintervention $(\mathrm{n}=795)$ & Postintervention $(\mathrm{n}=776)$ & $P$ Value \\
\hline Maternal age (years) & $27.5(5.6)$ & $28.0(5.6)$ & $.92^{\dagger}$ \\
\hline Race & & & $<.01^{\ddagger}$ \\
\hline Asian & $6.3(50)$ & $6.1(47)$ & \\
\hline African American & $19.3(153)$ & $19.2(148)$ & \\
\hline Hispanic & $26.2(208)$ & $40.3(311)$ & \\
\hline Native American & $0.9(7)$ & $1.4(11)$ & \\
\hline Caucasian & $47.4(377)$ & $32.9(254)$ & \\
\hline Pre-BMI & & & $.06^{\ddagger}$ \\
\hline$<18.5$ (Underweight) & $4.8(38)$ & $3.2(25)$ & \\
\hline 18.5 to 24.9 (Normal) & $40.0(318)$ & $36.6(283)$ & \\
\hline 25.0 to 29.9 (Overweight) & $28.2(224)$ & $27.7(214)$ & \\
\hline$>30.0$ (Obese) & $27.0(215)$ & $32.5(252)$ & \\
\hline Insurance & & & $.86^{\ddagger}$ \\
\hline Commercial & $29.3(230)$ & $28.5(221)$ & \\
\hline Medicaid & $69.3(544)$ & $69.9(542)$ & \\
\hline Self-pay & $1.4(11)$ & $1.7(13)$ & \\
\hline Parity & & & $.74^{\ddagger}$ \\
\hline 0 & $35.4(281)$ & $34.7(270)$ & \\
\hline$>1$ & $64.6(514)$ & $65.3(506)$ & \\
\hline
\end{tabular}

BMI, body mass index.

*Values are presented as \% $(\mathrm{N})$ for categorical and mean (SD) for continuous.

${ }^{\dagger}$ From Student's $t$ test.

${ }^{\ddagger}$ From Pearson's $\chi^{2}$ test of proportions. 
Table 3. Primary and Secondary Outcomes by Group*

\begin{tabular}{|c|c|c|c|}
\hline Variable & Pre-Intervention $(\mathrm{n}=795)$ & Post-Intervention $(\mathrm{n}=776)$ & p-Value \\
\hline IOM counseling & & & $<0.01^{\ddagger}$ \\
\hline No & $89.9(715)$ & $37.2(289)$ & \\
\hline Yes & $10.1(80)$ & $62.8(487)$ & \\
\hline Total weight gain (lb) & $28.3(13.9)$ & $25.9(13.7)$ & $.61^{\dagger}$ \\
\hline IOM weight gain (lb) & & & $.10^{\ddagger}$ \\
\hline Low & $22.01(175)$ & $25.77(200)$ & \\
\hline Recommended & $31.57(251)$ & $32.73(254)$ & \\
\hline High & $46.42(369)$ & $41.49(322)$ & \\
\hline Gestational age (weeks) & & & $.13^{\ddagger}$ \\
\hline$<37$ & $4.7(37)$ & $2.8(22)$ & \\
\hline$>37$ and $<41$ & $85.7(681)$ & $86.2(667)$ & \\
\hline$>41$ & $9.7(77)$ & $11.0(85)$ & \\
\hline Birthweight (g) & & & $.68^{\ddagger}$ \\
\hline$<2500$ & $2.6(21)$ & $2.2(17)$ & \\
\hline 2500 to 4000 & $89.3(709)$ & $90.6(703)$ & \\
\hline$>4000$ & $8.1(64)$ & $7.2(56)$ & \\
\hline Route of delivery & & & $.39^{\ddagger}$ \\
\hline Spontaneous vaginal & $76.9(611)$ & $77.8(601)$ & \\
\hline Assisted vaginal & $4.8(38)$ & $4.1(32)$ & \\
\hline Primary cesarean & $11.8(94)$ & $10.0(77)$ & \\
\hline Repeat cesarean & $6.5(52)$ & $8.1(63)$ & \\
\hline Hypertension & & & $.61^{\ddagger}$ \\
\hline No & $92.2(733)$ & $91.5(710)$ & \\
\hline Yes & $7.8(62)$ & $8.5(66)$ & \\
\hline Gestational diabetes & & & $<.01^{\ddagger}$ \\
\hline No & $88.5(530)$ & $92.7(719)$ & \\
\hline Yes & $11.5(69)^{\S}$ & $7.3(57)^{\|}$ & \\
\hline Shoulder dystocia & & & $.01^{\ddagger}$ \\
\hline No & $94.4(607)$ & $97.2(617)$ & \\
\hline Yes & $5.6(26)$ & $2.8(18)$ & \\
\hline Sensitivity analysis, yes ${ }^{\pi}$ & $3.1(19)$ & $2.4(16)$ & $.22^{\ddagger}$ \\
\hline
\end{tabular}

IOM, Institute of Medicine.

${ }^{*}$ Values are presented as \% (N) for categorical and mean (SD) for continuous.

${ }^{\dagger}$ From Student's t test.

${ }^{\ddagger}$ From Pearson's $\chi^{2}$ test of proportions.

${ }^{\S}$ Missing values from one site $(69 / 599=11.5 \%)$.

"If exclude site missing pre-data, post-intervention gestational Diabetes $=7.6 \%$. No significant difference.

${ }^{\pi}$ Omitting site that changed diagnostic criteria between pre- and postintervention time period.

No significant difference was identified between pre- versus postintervention regarding shoulder dystocia after sensitivity analysis revealed that 1 site changed their diagnostic criteria between the preand postintervention time periods. Therefore, data from that site were excluded (Table 3). Specifically, this 1 site overreported shoulder dystocia (in the preintervention phase only) as any use of the McRoberts maneuver. The consistent consensus among our other 6 sites was that shoulder dystocia was indicated if more techniques than the McRoberts maneuver were needed to accomplish vaginal delivery (e.g., suprapubic pressure, Rubin or Woods rotations, or removing the posterior fetal arm.).

No significant difference was identified between pre- and postintervention groups and the incidence of low birth weight $(<2500 \mathrm{~g}): 2.6 \%$ of those in the preintervention group having a low birth weight compared with $2.2 \%$ of those in the postintervention group $(P=.68$; Table 3$)$. There were also no significant differences in gestational age, macrosomia (birth weight, $>4 \mathrm{~kg}$ ), route of delivery, and incidence of hypertensive disorders (Table 3). 


\section{Discussion}

By improving weight gain counseling in accordance with the IOM 2009 guideline, we were successful in documenting a decrease in the incidence of gestational diabetes (number needed to treat $=24$. There were no significant decreases in macrosomia, postdate pregnancy, primary cesarean delivery, hypertensive disorders, and shoulder dystocia.

Our data confirmed the known associations ${ }^{2,5}$ between increased prepregnancy BMI and increased hypertensive disorders, gestational diabetes, birth weight of $>4 \mathrm{~kg}$, and primary cesarean (data not shown). Our influence on the incidence of gestational diabetes agrees with recent evidence: in a 2017 meta-analysis, dietary advice showed some effectiveness in reducing gestational diabetes $(\mathrm{RR}=$ $0.60 ; 95 \%$ CI, $0.35-1.04 ; 5$ trials; $\mathrm{n}=1279$; $P=.07) .{ }^{14}$ In addition, in agreement with our results, others did not show clear decreases in fetal macrosomia, cesarean section, nor preeclampsia. ${ }^{15} \mathrm{We}$ speculate that this is due to the powerful effect of prepregnancy BMI on these outcomes. Our intervention did not target changing prepregnancy BMI.

Despite the increased BMI among the mothers who presented for care in 2015-2017, we were still able to show a decrease in the incidence of gestational diabetes with improved weight gain counseling. We hypothesize this is due to more mothers modifying their eating and activity before gestational diabetes screening at 24 weeks of gestation and, hence, more passing the screen as "normal." Clinicians and policymakers can be more confident that time spent in prenatal counseling will be beneficial. The IOM Pregnancy Weight Gain Guideline has been validated by this study and others. ${ }^{14,16}$ Hopefully our decrease in gestational diabetes will translate into less type 2 diabetes and fewer complications later in the lives of our mothers.

Additional research is needed to discover and validate effective prenatal clinical interventions to mitigate the strong influence of prepregnancy BMI on obstetric outcomes. Weight gain counseling before pregnancy at family planning, primary care, and postpartum medical visits might be fruitful episodes of care for new interventions.

\section{Strengths and Limitations}

Strengths of this study include its real world, clinical approach, its roots in motivational inter- viewing, and the brevity of this type of educational intervention.

Limitations of our study include the pre/postintervention design, rather than a contemporaneous randomized trial. However, by the time of our intervention (2014) it would have been unethical to randomize care sites to neglect the IOM expert opinion clinical guideline that was published in 2009. Our major finding of reduced gestational diabetes could be due to some factor that changed between preintervention (2012-13) and postintervention (2015-17) time periods other than our educational intervention to improve weight gain counseling. Our multiple regression analysis indicated this was not likely: there was no significant association between demographic variables at entry to prenatal care and pregnancy outcomes in pre- versus postintervention groups. A per protocol analysis was also confirmatory.

A second limitation was that the type, intensity, timing, and recording of prenatal weight gain counseling were variable across individual sites and providers. To investigate timing, in the postintervention period, we added data collection (at 6 of 7 sites) of whether weight gain counseling began before 20 weeks of gestation. That was the case in $89.7 \%$ of postintervention mothers. This was to ensure enough time was available for the counseling to have an influence. If few of the mothers had been counseled before 20 weeks, it would have been harder to argue that the counseling itself made any difference in the outcomes. The documentation of weight gain counseling may also have been influenced by the availability of electronic medical record templates or prompts at some sites but not at others. There is the possibility that weight gain counseling was performed for some patients but not clearly documented in their prenatal record and was, hence, misclassified as "not done" in our data reporting.

\section{Conclusions}

Improving weight gain counseling is an achievable goal and our data indicate it has promise in improving pregnancy outcomes-specifically a reduction in the incidence of gestational diabetes.

Significant assistance was provided by Patricia Corona, medical student; John Halerz, MD; Micah Hong, DO; Cristina Lammers, MD, MPH; Gloria Okereke, DO; Jahanvi Patel, DO; and Erika Sanders, MD. Study Site Principal Investigators: 
Alicia Milan-Flannigan, MD, St. Mary's Medical Center Family Medicine, Chicago, IL; Claire Philippe, MD, Leslie Sleuwen, MD, Hinsdale Family Medicine, Hinsdale, IL; Sanjjini Thomas, MD, Resurrection Medical Center, Chicago, IL; Kwanza Devlin, MD, University of North Dakota, Fargo, ND; Nicky Eustace, MD, University of Illinois, Dixon, IL.

To see this article online, please go to: http://jabfm.org/content/ 33/2/189.full.

\section{References}

1. Stotland NE, Gilbert P, Bogetz A, Harper CC, Abrams B, Gerbert B. Preventing excessive weight gain in pregnancy: how do prenatal care providers approach counseling? J Women's Health 2010;19: 807-14.

2. Johnson J, Clifton RG, Roberts JM, et al. Pregnancy outcomes with weight gain above or below the 2009 Institute of Medicine guidelines. Obstet Gynecol 2013;121:969-75.

3. Rasmussen KM, Yaktine AL, Committee to Reexamine IOM Pregnancy Weight Guidelines. Food and Nutrition Board, Board on Children, Youth and Families, Institute of Medicine, National Research Council. Weight gain during pregnancy: reexamining the guidelines. Washington DC: The National Academies Press; 2009.

4. Schummers L, Hutcheon JA, Bodnar LM, Lieberman E, Himes KP. Risk of adverse pregnancy outcomes by prepregnancy body mass index: a population-based study to inform prepregnancy weight loss counseling. Obstet Gynecol 2015;125:133-43.

5. Wei YM, Yang HX, Zhu WW, et al. Risk of adverse pregnancy outcomes stratified for pre-pregnancy body mass index. J Matern Fetal Neonatal Med 2016;29:2205-9.

6. Goldstein RF, Abell SK, Ranasinha S, et al. Association of gestational weight gain with maternal and infant outcomes: a systematic review and metanalysis. JAMA 2017;317:2207-25.

7. McDonald SD, Pullenayegum E, Taylor VH, et al. Despite 2009 guidelines, few women report being counseled correctly about weight gain during pregnancy. Am J Obstet Gynecol 2011; 205:333.e1-6.

8. Muktabhant B, Lumbiganon P, Ngamjarus C, Dowswell T. Interventions for preventing excessive weight gain during pregnancy. Cochrane Database Syst Rev 2012;4:CD007145.

9. Sanabria-Martínez G, García-Hermoso A, PoyatosLeón R, Álvarez-Bueno C, Sánchez-López M, Martínez-Vizcaíno V. Effectiveness of physical activity interventions on preventing gestational diabetes mellitus and excessive maternal weight gain: a meta-analysis. BJOG: International J Obstet Gynaecol 2015;122:1167-74.

10. Stafne SN, Salvesen KĐ, Romundstad PR, Eggebø TM, Carlsen SM, Mørkved S. Regular exercise during pregnancy to prevent gestational diabetes: a randomized controlled trial. Obstet Gynecol 2012; 119:29-36.

11. American College of Obstetricians and Gynecologists. Gestational diabetes mellitus. Practice Bulletin number 137. Obstet Gynecol 2013;122:406-16.

12. American College of Obstetricians and Gynecologists. Hypertension in pregnancy. Report of the American college of obstetricians and gynecologists' task force on hypertension in pregnancy. Obstet Gynecol 2013; 122:1122.

13. Eve Ackerman DNP, Falsetti SA, Lewis P, Hawkins AO, Heinschel JA. Motivational interviewing: a behavioral counseling intervention for the family medicine provider. Fam Med 2011;43:582-5.

14. Tieu J, Shepherd E, Middleton P, Crowther CA. Dietary advice interventions in pregnancy for preventing diabetes mellitus. Cochrane Database Syst Rev 2017;1:CD006674.

15. Muktabhant B, Lawrie TA, Lumbiganon $P$, Laopaiboon M. Diet or exercise, or both, for preventing excess weight gain in pregnancy. Cochrane Database Syst Rev 2015;6:CD007145.

16. LifeCycle Project-Maternal Obesity and Childhood Outcomes Study Group. Association of gestational weight gain with adverse maternal and infant outcomes. JAMA 2019;321:1702-15. 


\section{Appendix 1: Provider \& Patient Handouts}

Clinician's Tool (2 pages)

Patient Education: Initial Brochure (2 pages)

Patient's Motivations (1 page)

Patient education handout: "How much extra each day?" (1 page)

Provider and patient education handouts will be available online at: https://www.advocatehealth.com/ education/residency-opportunities/advocate-christ-medical-center/family-medicine/.

\section{Appendix 2}

Table 4. Multivariate Regression Models for Weight Gain* $(\mathrm{n}=1571)$

\begin{tabular}{|c|c|c|}
\hline Variable & Low Weight Gain & High Weight Gain \\
\hline Intervention group & $1.20(0.85-1.69) ; P=.31$ & $0.85(0.63-1.16) ; P=.10$ \\
\hline IOM Counseling & $0.83(0.99-1.04) ; P=.30$ & $1.04(0.76-1.43) ; P=.79$ \\
\hline \multicolumn{3}{|l|}{ Site } \\
\hline $1 \mathrm{UIC}$ & Reference & Reference \\
\hline 2 StMary's & $1.02(0.64-1.62) ; P=.46$ & $0.76(0.50-1.17) ; P=.12$ \\
\hline 3 Hinsdale & $0.68(0.42-1.10) ; P=.12$ & $1.06(0.70-1.59) ; P=.60$ \\
\hline 4 Adv Christ & $1.06(0.66-1.70) ; P=.31$ & $1.07(0.71-1.61) ; P=.57$ \\
\hline 5 Resurrection & $0.74(0.40-1.37) ; P=.43$ & $0.69(0.40-1.19) ; P=.09$ \\
\hline $6 \mathrm{~N}$ Dakota & $0.86(0.42-1.78) ; P=.90$ & $1.12(0.63-2.01) ; P=.56$ \\
\hline 7 Rockford, IL & $0.96(0.44-2.09) ; P=.81$ & 1.30 (0.69-2.44); $P=.25$ \\
\hline \multicolumn{3}{|l|}{ Race } \\
\hline Caucasian & Reference & Reference \\
\hline Asian & $1.01(0.55-1.83) ; P=.15$ & $0.55(0.31-0.97) ; P=.04$ \\
\hline African American & $0.96(0.62-1.48) ; P=.05$ & $0.72(0.49-1.05) ; P=.16$ \\
\hline Hispanic & $0.94(0.64-1.37) ; P=.04$ & $0.72(0.52-1.02) ; P=.15$ \\
\hline Native American & $11.42(1.40$ to 93.30$) ; P=.02$ & $4.03(0.48-34.17) ; P=.12$ \\
\hline \multicolumn{3}{|l|}{ Pre-BMI } \\
\hline$<18.5$ (Underweight) & $0.98(0.54-1.80) ; P=.76$ & $0.35(0.16-0.74) ; P<.01$ \\
\hline 18.5 to 24.9 (Normal) & Reference & Reference \\
\hline 25.0 to 29.9 (Overweight) & $0.64(0.44-0.94) ; P=.02$ & $2.16(1.60-2.93) ; P<.01$ \\
\hline$>30.0$ (Obese) & $1.11(0.78-1.57) ; P=.17$ & $2.25(1.64-3.08) ; P<.01$ \\
\hline \multicolumn{3}{|l|}{ Insurance } \\
\hline Commercial & Reference & Reference \\
\hline Medicaid & $1.52(1.07-2.16) ; P=.86$ & $1.25(0.92-1.69) ; P=.88$ \\
\hline Self-pay & $2.58(0.81-8.27) ; P=.21$ & $1.42(0.47-4.27) ; P=.67$ \\
\hline Parity & $1.02(0.73-1.43) ; P=.91$ & $0.54(0.41-0.73) ; P<.01$ \\
\hline Maternal age & $1.01(0.98-1.03) ; P=.60$ & $0.98(0.96-1.01) ; P=.16$ \\
\hline
\end{tabular}

IOM, Institute of Medicine; BMI, body mass index; UIC, University of Illinois, Chicago.

${ }^{*}$ Values are presented as odds ratio $(95 \% \mathrm{CI})$. 
Table 5. Multivariate Regression Models for Secondary Outcomes* $(n=1571)$

\begin{tabular}{|c|c|c|}
\hline Variable & Gestational Diabetes & Shoulder Dystocia \\
\hline Intervention group & $0.54(0.32-0.91) ; P=.02$ & $0.65(0.33-1.30) ; P=.22$ \\
\hline IOM Counseling & $0.94(0.56-1.58) ; P=.81$ & $0.31(0.12-0.78) ; P=.01$ \\
\hline \multicolumn{3}{|l|}{ Site } \\
\hline $1 \mathrm{UIC}$ & Reference & Reference \\
\hline 2 StMary's & $1.13(0.51-2.52) ; P=.77$ & $0.94(0.35-2.56) ; P=.35$ \\
\hline 3 Hinsdale & $1.72(0.77-3.85) ; P=.11$ & $1.86(0.77-4.51) ; P<.01$ \\
\hline 4 Adv Christ & $1.12(0.49-2.57) ; P=.74$ & $0.49(0.16-1.55) ; P=.54$ \\
\hline 5 Resurrection & $0.61(0.20-1.87) ; P=.08$ & $0.19(0.02-1.59) ; P=.16$ \\
\hline 6 N Dakota & $2.67(1.06-6.69) ; P<.01$ & $0.50(0.12-2.15) ; P=.67$ \\
\hline 7 Rockford, IL & $1.10(0.35-3.47) ; P=.80$ & $0.57(0.13-2.45) ; P=.82$ \\
\hline \multicolumn{3}{|l|}{ Race } \\
\hline Caucasian & Reference & Reference \\
\hline Asian & $4.50(1.99-10.20) ; P<.01$ & $0.26(0.03-1.99) ; P=.98$ \\
\hline African American & $0.65(0.30-1.39) ; P=.02$ & $0.77(0.32-1.84) ; P=.96$ \\
\hline Hispanic & $2.10(1.25-3.54) ; P=.11$ & $0.71(0.33-1.56) ; P=.96$ \\
\hline Native American & $0.94(0.19-4.68) ; P=.53$ & $<0.01(<0.01->999.99) ; P=.97$ \\
\hline \multicolumn{3}{|l|}{ Pre-BMI } \\
\hline$<18.5$ (Underweight) & $0.53(0.11-2.46) ; P=.11$ & $0.88(0.19-4.04) ; P=.68$ \\
\hline 18.5 to 24.9 (Normal) & Reference & Reference \\
\hline 25.0 to 29.9 (Overweight) & $1.84(1.08-3.16) ; P=.20$ & $1.38(0.69-2.77) ; P=.45$ \\
\hline$>30.0$ (Obese) & $3.32(1.98-5.57) ; P<.01$ & $1.26(0.59-2.69) ; P=.68$ \\
\hline \multicolumn{3}{|l|}{ Insurance } \\
\hline Commercial & Reference & Reference \\
\hline Medicaid & $0.51(0.11-2.46) ; P=.76$ & $1.44(0.66-3.15) ; P=.32$ \\
\hline Self-pay & $0.34(0.07-1.67) ; P=.36$ & $5.50(0.97-31.11) ; P=.07$ \\
\hline Parity & $1.16(0.70-1.91) ; P=.57$ & $1.30(0.64-2.64) ; P=.47$ \\
\hline Maternal age & $1.07(1.03-1.11) ; P<.01$ & $0.98(0.92-1.04) ; P=.45$ \\
\hline
\end{tabular}

IOM, Institute of Medicine; BMI, body mass index; UIC, University of Illinois, Chicago.

${ }^{*}$ Values are presented as odds ratio $(95 \% \mathrm{CI})$. 